THE ROUTINE MANAGEMENT OF SYPHILIS AND GONORRHEA EMPLOYED IN THE ST. THOMAS'S HOSPITAL VENEREAL DISEASES DEPARTMENT

By L. W. HARRISON, D.S.O., M.B., F.R.C.P.E., Brevet-Colonel R.A.M.C., and K.H.P. (Ret.), Director.

\title{
I
}

THE greater part of the following sketch of the " Routine Treatment of Syphilis and Gonorrhœea " employed in the Venereal Diseases Department at St. Thomas's Hospital appeared in vol. xxix. of St. Thomas's Hospital Gazette. The demand for reprints has been so great that it has seemed advisable to revise the original paper and bring it up to date. I am indebted to the editor of St. Thomas's Hospital Gazette for permission to reproduce here so much of the original article.

The following is a bare statement of the lines of treatment usually followed, unburdened by any long discussion of reasons for the various forms of treatment outlined, since most of these can be found in my works on the subject. To prevent any misconception which might arise from the use of the word " routine" in the title of this paper, it should be said at once that it does not mean that a programme of treatment is carried out mechanically. Indeed, this is far from being the case : every patient is carefully examined before each further step is taken, and the treatment is modified if the circumstances of the case require it.

It is convenient to divide the routine into that employed for males and females respectively in the case of each disease. The following is the programme for

\section{SyPhilis in Males}

Great pains are taken to insure a certain diagnosis as early as possible, especially in the case of genital sores. Specimens for microscopical examination by the darkground method are taken from all sores, and, in the event of failure to find $S p$. pallida in the serum exudate from 


\section{BRITISH JOURNAL OF VENEREAL DISEASES}

these, specimens are taken from any neighbouring glands which may be enlarged.

Although the clinical appearances and laboratory tests may indicate a diagnosis of chancroid, syphilis is not finally excluded until all tests for this disease have proved negative for three months.

For treatment, the remedies employed are arsenobenzol compounds, mercury, bismuth and potassium iodide.

The Arsenobenzol Compounds used are divisible into a number of classes as follows :-

(I) Preparations which are chemically dioxydiaminoarseno-benzol-monomethylene-sulphoxylate of soda, to which Ehrlich originally gave the number " 9I4." They are now sold in this country under a variety of trade names, including neokharsivan, neosalvarsan, novarsenobillon and novarsan.

(2) A compound which is similar to " $9 \mathrm{I} 4$ " and sold as sulfarsenol. Besides sulfarsenol there are now other compounds, such as kharsulphan, of similar chemical constitution. They are employed in this department exclusively for deep subcutaneous or intramuscular injection, because I do not think them so effective as the others when given intravenously, but they cause less local discomfort when injected into the tissues, and, I think, act then better than the other preparations.

(3) A combination of the sodium compound of dioxydiaminoarsenobenzol (sodium-salvarsan) with colloidal silver and known as silver-salvarsan. Another preparation of similar nature which is now again on the market is arseno-argenticum.

(4) A compound of dioxydiaminoarsenobenzol, which is the base of the arsenobenzol compounds (No. 592 in the Ehrlich series), with glucose and sold under the name of stabilarsan.

The Mercurial Preparations are Io or 20 per cent. mercurial cream for intramuscular injection, and hydrarg. c. cret. or hydrarg. iodid. flav. for administration by the mouth-the former sometimes in combination with Dover's powder.

Intramuscular injections were formerly given in preference to treatment by mouth, but have now been superseded by injections, generally deep subcutaneous, of bismuth.

Many Bismuth Preparations, sold under a number of 


\section{MANAGEMENT OF SYPHILIS AND GONORRHGA}

trade names, have been tested, and the results agree generally with those recorded in the literature, but it is impossible yet to say which is most suitable, or to indicate exactly in what respects they should be employed to replace other anti-syphilitic remedies. Generally, in the doses which can be employed, they are more active than mercurial compounds, but less so than the arsenobenzol. When given in greater doses than the patient can tolerate they cause much the same toxic effects as do mercurial preparations-namely, stomatitis and albuminuria.

Of the very great number of preparations now on the market, those employed most commonly in the department are suspensions in glucose solution of precipitated bismuth and of bismuth oxychloride. The watery suspension is preferred to the oily, since, as Lomholt has shown, they are absorbed more rapidly and evenly than these. The weekly dose is from 0.2 to 0.4 gramme of the metal, and the dose is placed under the fat and over the fascia covering the gluteal region, since this choice of site reduces to a minimum the chances of the remedy being injected into a vessel.

\ Potassium Iodide is employed to stimulate resolution of infiltrates and so prepare the ground for further attacks on $S p$. pallida.

In the following tables the arsenobenzol preparations are alternative, and mercury or bismuth is additional to the arsenobenzol, being administered on the same days as the arsenobenzol, except when contra-indicated by

N) signs of mercurial or bismuth poisoning, or when silver-

* salvarsan is given. The explanation of the abbreviations 5 employed in the tables is as follows :-

"Day" and the numbers under it mean the first, eighth, and so on, days on which the patient is treated.

"9I4" means any of the preparations mentioned under (I) in the text above, injected intravenously in a Io per cent. solution.

"S.S." means silver-salvarsan injected intravenously in a I per cent. solution.

"Stab." means stabilarsan injected intravenously as taken from the ampoule.

"Sulf." means sulfarsenol, or similar preparation, dissolved in $I$ to 2 c.c. water and injected deep subcutaneously-that is, on the surface of the fascia covering the gluteal muscles, not into the muscle or the fat. 


\section{BRITISH JOURNAL OF VENEREAL DISEASES}

"Hg." means mercury in a ro or 20 per cent. suspension.

"Bi." means one of the bismuth preparations in a Io or 20 per cent. suspension or solution.

"Grm." means grammes of the dried compound except in the case of stabilarsan, as explained below.

" Gr." means grains of the dried compound.

The patient receives a card of simple instructions as to his mode of life. It advises him to eat plenty of bread, jam, milk puddings, vegetables and fruit ; not so much meat, fish and fat, and no fried fish or shell fish ; to drink plenty of plain water and no patent medicines; to smoke little, avoid alcohol, take much outdoor exercise, and dress warmly.

Before each injection every patient's skin and mucous membranes are examined for signs of intolerance in the form of erythema or jaundice, and questions are asked to ascertain if the patient is experiencing such toxic effects as general malaise, sleeplessness, loss of appetite, or severe reaction after each successive injection. The injections of arsenobenzol are stopped in the event of jaundice or erythema of the smallest degree, and the doses may be reduced for minor signs of intolerance. The urine is always examined for albumen, and the dosage may be modified in the event of this appearing likely to be aggravated by the treatment. Before each arsenobenzol injection the patient is made to drink the following :-

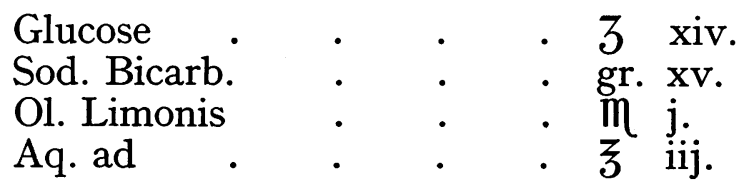

This is in the hope that the liver cells will become filled with glycogen and have less affinity for the arsenobenzol, which is a liver-cell poison and may cause jaundice. For the same reason patients are not encouraged to starve for long hours before the injection, as is often recommended. Two hours' abstinence seems to be sufficient preparation for an intravenous injection, and no abstinence at all is required before a subcutaneous.

It is necessary to mention some precautions which are taken in the technique of the injections as they affect the incidence of toxic reactions. The syringe and needle are washed free of all spirit by filling with distilled water and 
emptying; this is particularly important when using stabilarsan. In the case of silver-salvarsan and stabilarsan, also, care is taken that the vein wall is not impregnated with the solution, by seeing that no bead of this is at the end of the needle when it is inserted, and by drawing a little blood back into the syringe when the solution has been injected. This precaution is to prevent thrombosis of the vessel, which is troublesome, though not a disaster. Stabilarsan and silver-salvarsan must be injected particularly slowly. This and the precaution against contamination of the solution with spirit are to prevent vaso-dilator symptoms.

In the case of patients who are particularly prone to suffer from vaso-dilator symptoms the plan of injection recommended by Sicard is adopted. The tourniquet is retained during the injection and for some minutes afterwards, a pad being pressed firmly on the puncture as soon as the needle has been withdrawn.

The various courses may conveniently be divided into four classes, A, B, C, and D, and the dosage indicated is that for adult males of average weight.

In Course A from the fifty-eighth to the seventyseventh day potassium iodide is given in doses of 5 grains thrice daily the first week; 7 grains the second week; and Io grains the third week, generally in combination with nux vomica. A blood test is taken on the ninetysecond day.

Course A

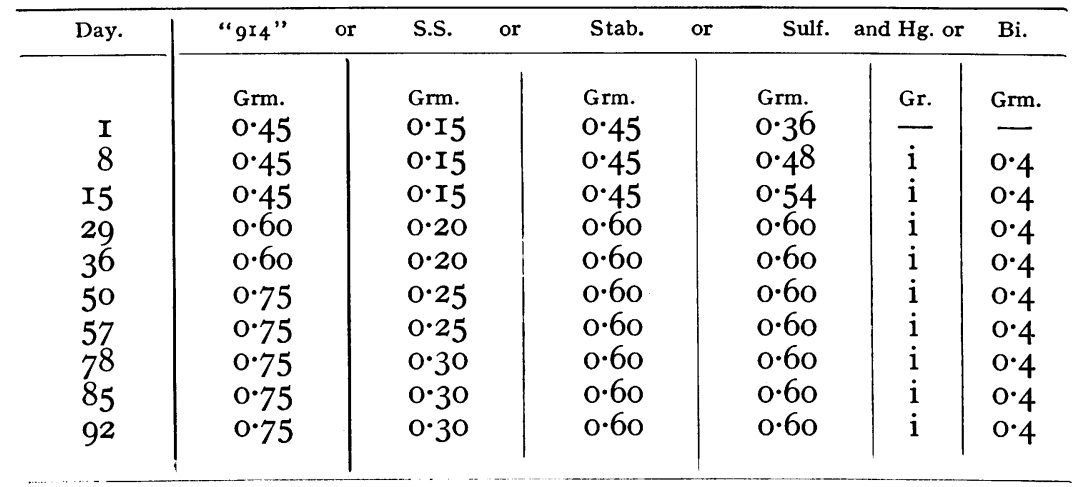

Course B

The same as Course A but omitting the last three injections, from the seventy-eighth to the ninety-second day. 
BRITISH JOURNAL OF VENEREAL DISEASES

Course $\mathrm{C}$

\begin{tabular}{|c|c|c|c|c|c|c|c|c|c|}
\hline Day. & “914" & or & s.s. & or & Stab. & or & Sulf. & and $\mathrm{Hg}$. or & Bi. \\
\hline & Grm. & & Grm. & & Grm. & & Grm. & Gr. & Grm. \\
\hline $\begin{array}{l}1 \\
8\end{array}$ & $\begin{array}{l}0.60 \\
0.60\end{array}$ & & $\begin{array}{l}0.20 \\
0.20\end{array}$ & & $\begin{array}{l}0.45 \\
0.60\end{array}$ & & $\begin{array}{l}0.54 \\
0.60\end{array}$ & $\begin{array}{l}1 \\
\mathrm{i}\end{array}$ & $\begin{array}{l}0.4 \\
0.4\end{array}$ \\
\hline I5 & 0.60 & & 0.20 & & 0.60 & & 0.60 & $\mathrm{i}$ & 0.4 \\
\hline 29 & 0.75 & & 0.30 & & 0.60 & & $0 \cdot 60$ & $\mathrm{i}$ & 0.4 \\
\hline 36 & 0.75 & & 0.30 & & $0 \cdot 60$ & & $0 \cdot 60$ & $\mathrm{i}$ & 0.4 \\
\hline
\end{tabular}

Notes on THE Courses

(I) The doses of the alternative arsenobenzol preparations do not correspond with one another according to arsenical content, but have been fixed in the case of each preparation partly according to the tolerance of the average patient and partly according to the therapeutic effect. Thus silver-salvarsan in a dose of $0 \cdot I 5$ gramme has about the same therapeutic effect on animals as 0.45 gramme " 9I4," but in the later stages of the course we often increase the dose to 0.30 , which is equivalent to 0.9 of " 9I4," partly because it is well tolerated in this dose, and partly because, as mentioned below, mercury or bismuth is not administered at the same time as silversalvarsan. The dose indicated on the ampoule of stabilarsan does not express the weight of dried remedy but the equivalent of "9I4" as judged by arsenical content, but we have reason to believe that it is retained longer

COURSE D

\begin{tabular}{|c|c|c|c|c|c|c|c|}
\hline Day. & S.S. & or & Stab. & or & Sulf. & and $\mathrm{Hg}$. or & Bi. \\
\hline$I$ & Grm. & & $\begin{array}{l}\text { Grm. } \\
0 \cdot 30\end{array}$ & & $\begin{array}{c}\text { Grm. } \\
0.36\end{array}$ & Gr. & Grm. \\
\hline 4 & O.I5 & & $0 \cdot 30$ & & $0 \cdot 36$ & $\mathrm{i}$ & 0.2 \\
\hline 8 & O.I5 & & $0 \cdot 30$ & & $0 \cdot 36$ & - & 0.2 \\
\hline II & $0 \cdot I_{5}$ & & $0 \cdot 30$ & & $0 \cdot 36$ & $\mathrm{i}$ & 0.2 \\
\hline I5 & 0.20 & & 0.45 & & 0.48 & - & 0.2 \\
\hline I8 & $0 \cdot 20$ & & 0.45 & & $0 \cdot 48$ & i & 0.2 \\
\hline 22 & $0 \cdot 20$ & & 0.45 & & $0 \cdot 48$ & 一 & 0.2 \\
\hline 25 & $0 \cdot 20$ & & - & & - & $\mathrm{i}$ & $0 \cdot 2$ \\
\hline 29 & $0 \cdot 20$ & & 0.60 & & 0.60 & - & 0.2 \\
\hline 32 & $0 \cdot 25$ & & - & & - & i & 0.2 \\
\hline 36 & $0 \cdot 25$ & & $0 \cdot 60$ & & $0 \cdot 60$ & - & 0.2 \\
\hline 39 & 0.25 & & - & & - & $\mathrm{i}$ & 0.2 \\
\hline 43 & 0.25 & & 0.60 & & 0.60 & - & 0.2 \\
\hline
\end{tabular}




\section{MANAGEMENT OF SYPHILIS AND GONORRHCEA}

in the body and has a greater cumulative effect ; for this reason it has been thought advisable not to increase beyond 0.6 gramme. Sulfarsenol corresponds fairly closely in arsenical content to " 9I4," but it is administered subcutaneously, and the first dose is kept low lest the patient be one who reacts severely at the site of injection and fail to return for treatment. The dose of 0.60 is not exceeded, so that local reaction may not be too severe and because the therapeutic effect of an arsenobenzol preparation is greater when it is given subcutaneously than when it is given intravenously.

(2) Silver-salvarsan is included in the tables above as a matter of convenience, but when the patient is treated throughout with this remedy, mercury or bismuth is deferred to the intervals between the courses of arsenobenzol.

(3) As a general rule, the same arsenobenzol preparation is used throughout a single course of treatment of a given patient, but this is largely a matter of convenience in the routine of managing large numbers of cases, and when it seems better to change from one preparation to another, this is done. At the present moment some observations on stabilarsan point to the desirability of changing over to this remedy as a matter of routine after the patient has received a few doses of one of the other preparations. The reason is that stabilarsan does not act quickly as tested by the disappearance of spirochætes from the serum of superficial lesions, but, in corresponding doses, has eventually a greater therapeutic effect than "9I4" as shown by the serum tests. If it is decided to treat by intravenous injection, not using silver-salvarsan, the course for early cases which is indicated by this observation would commence with about three doses of "9I4," to render the patient non-infective as quickly as possible, and would continue with stabilarsan intravenously for the sake of its more profound effect in the long run. Sulfarsenol is usually chosen for patients who are apt to react with vaso-dilator symptoms when injected intravenously. I prefer it and similar compounds which can be given subcutaneously to all other arsenobenzol preparations for the treatment of syphilis of the heart and aorta. Patients suffering from syphilitic myocarditis seem to be particularly prone to suffer from cardiac disturbance if the arsenobenzol enters the circulation in too great 


\section{BRITISH JOURNAL OF VENEREAL DISEASES}

concentration, and not only has the remedy to be injected subcutaneously, but great care must be taken over any increase of dosage to more than 0.30 gramme. It is eminently suitable for use throughout the course in every case, and has the advantage that the patient may eat a meal immediately before the injection, whereas, under these conditions, an intravenous injection is an almost certain emetic. Sulfarsenol would be used much more largely than it is if it were not for the fact that it may cause some local pain, and many patients are so foolish as to discontinue attendance if the injections cause them any discomfort. Silver-salvarsan is not quite so convenient to administer as the others and is rather apt to cause vaso-dilator symptoms, but has been used extensively in the department, especially for nerve cases, for which it seems to be very suitable. It is a common practice to change over to a different arsenobenzol preparation when the patient returns for a second or subsequent course of treatment.

(4) Mercury, or bismuth, is usually administered at the same time as arsenobenzol, partly for the sake of convenience, but chiefly because I believe that the two act better when given simultaneously than when they are administered in separate courses. This belief is supported by some animal experiments by Lehnhoff-Wyld, which showed that the action of arsenobenzol is intensified when another metal is present in the circulation at the same time.

Using the courses outlined above, with the modifications indicated in the notes, the following are the plans on which patients in different stages of infection are treated :-

\section{I}

Primary Cases with Negative Serum Reaction at TIME OF First INJECTION AND ONE WeEk AFTER THIS

(a) Course A.

(b) No treatment for five weeks.

(c) Potassium iodide for three weeks (gr. I5 a day for the first week, gr. 2I a day for the second, and gr. 30 for the third).

(d) Course A.

(e) Suspend all treatment, but test the blood serum every three months during the first year after stopping 


\section{MANAGEMENT OF SYPHILIS AND GONORRHEA}

treatment, and every six months during the second year; the test at the end of the first and second year respectively being preceded by a provocative injection of an arsenobenzol preparation, such as 0.45 gramme " 914 " or stabilarsan. The cerebro-spinal fluid is tested when the lumbar puncture is unlikely to interfere with the patient's work.

\section{II}

Primary Cases with a Positive Reaction at Time of First InJeCtion or ONE WeEk LATER

(a) As indicated in I $(a)$ to $(d)$.

(b) No treatment for ten weeks.

(c) Potassium iodide for three weeks.

(d) Course C.

(e) Suspend treatment and observe patient as indicated in I $(e)$ above.

\section{III}

Cases with Clinical Signs of Secondary Syphilis

(a) As shown in II $(a)$ to $(d)$.

(b) No treatment for ten weeks.

(c) Potassium iodide for three weeks.

(d) Course C.

(e) Suspend treatment and observe patient as indicated in $\mathrm{I}(e)$.

\section{IV}

Cases which are Later than Secondary

(a) Course B.

(b) Three weeks potassium iodide.

(c) No treatment for ten weeks.

(d) Course C.

(e) Three weeks potassium iodide.

(f) Repeat (c) to $(e)$ twice.

(g) No treatment for sixteen weeks.

(h) Course C.

(i) Three weeks potassium iodide.

(k) No treatment for eighteen weeks.

(l) Course C.

The results at St. Thomas's Hospital agree with those elsewhere in showing the great difficulty of converting a positive serum reaction in many of these cases to negative. 


\section{BRITISH JOURNAL OF VENEREAL DISEASES}

Some workers regard the task as hopeless, and would not continue treatment in the absence of active signs of syphilis. I would be glad to adopt this course if it could be proved that a positive serum reaction does not arise from spirochætal activity, or if I could learn how to tell when a syphilitic process is beginning to cause degenerations in heart, great vessels, central nervous system, or other important organs. But I can detect only the signs of damage when this has become considerable, and would therefore rather play on the embers than wait until the house is half burnt down. Those who would leave a patient untreated, because, as they say, he has only a positive serum reaction; who decry a policy of persistent treatment in these cases, stating that we have to treat patients, not reactions, appear to forget that syphilis is a disease not merely of the skin and visible mucous membranes. They have perhaps overlooked the fact that syphilis is, above all, a disease of blood vessels ; that, as shown by innumerable post-mortem records, a very high proportion of syphilitic patients have aortic disease ; that syphilitic aortic disease and myocarditis are silent as to signs and symptoms until the damage has become considerable and the patient more or less a wreck; and that too many men who have been thought healthy die suddenly of syphilitic heart disease which would never have reached this stage if the disease had been treated persistently.

\section{$\mathrm{V}$}

\section{Syphilis of the Nervous System}

(a) Course D, preferably employing bismuth.

(b) Three weeks potassium iodide.

(c) No treatment for three weeks.

(d) Repeat $(a)$ and $(b)$.

(e) No treatment for eight weeks.

(f) Repeat $(a)$ and $(b)$.

(g) No treatment for twelve weeks.

(h) Repeat $(a)$ and $(b)$.

(i) No treatment for fourteen weeks.

(k) Repeat $(a)$ and $(b)$.

The above treatment is controlled by periodical examinations of the cerebro-spinal fluid, and may be continued on the same lines for a number of years. 


\section{MANAGEMENT OF SYPHILIS AND GONORRHEA}

Cardio-vascular Syphilis.-For cardiac cases courses on the lines of those for syphilis of the nervous system are usually suitable, employing sulfarsenol and bismuth reducing the dosage of the arsenobenzol by at least 50 per cent. for the first four doses and by one-third for the remainder.

VI

Treatment of Certain Toxic Effects of Arsenobenzol

Severe vaso-motor disturbance is treated with a subcutaneous injection of adrenalin chloride (Io to I5 minims of I/IOOo). Measures for the prevention of this side effect have been mentioned above.

Cerebral symptoms with epileptiform convulsions would be treated by lumbar puncture, drawing off 20 to 30 c.c. fluid; bleeding to $\mathrm{I}$ pint, and injection of adrenalin chloride (I c.c. of I/IOOo solution diluted with 5 c.c. physiological saline given slowly into the muscles).

Erythema is often relieved by the first intravenous injection of 25 c.c. of a 25 per cent. solution of glucose made alkaline to litmus with caustic soda solution. This is repeated every day, or every other day, as long as required. More recently sodium thiosulphate has been employed in doses of 0.6 to 0.9 gramme, given intravenously or intramuscularly every day or every alternate day. Ampoules containing weighed amounts of pure sodium thiosulphate can be obtained from the manufacturing chemists. Exfoliative dermatitis is encountered very infrequently. It is treated as for erythema so long as the bend of the elbow is not involved. The patient is carefully nursed in bed. The diet is much the same as for cases of typhoid fever, with unlimited amounts of bland fluids, as much liquid carbohydrate food as the patient will take, and restricted proteins, fats and extractives. A recent case has seemed to be benefited by Marmite and cod-liver oil, given for the sake of their vitamines. In exfoliative dermatitis the digestive system is disturbed by a similar process to that which affects the skin, so that it is useless, and even harmful, to administer food which is not easily absorbed. As for local applications, the dry treatment appears to answer best, mainly consisting of the application of boric, zinc and starch powder and 


\section{BRITISH JOURNAL OF VENEREAL DISEASES}

calamine lotion, which is allowed to dry on the skin. Bran baths are reserved for times when the patient has become too uncomfortable from the exfoliation; which may be very severe. Such scales as will come off easily without leaving a raw surface are removed by hand. The principle of this treatment is that soaking and anointing the skin cause it to become macerated and give micro-organisms a better foothold and opportunity for incubation than when they rest on a dry surface which is fairly quickly shed. Oily applications are reserved strictly for parts where the skin has become fissured and painful. Throat symptoms, which may be very troublesome, are relieved by inhalations, and the mouth must be kept perfectly clean. The conjunctival sac must be kept free of pus by washing out frequently with a simple, antiseptic eye lotion.

After recovery it has been the custom not to repeat the arsenobenzol injections for at least a year. Further experience may prove, however, that the antidotal effects of sodium thiosulphate are sufficiently certain to permit of an earlier resumption of the arsenobenzol treatment.

Jaundice, when severe, is treated by intravenous injections of glucose as above, and by a similar diet to that employed for cases of dermatitis. The bowels are kept open as required, and a mixture of bismuth and sod. bicarb. is given.

\section{Syphilis in Females}

Females are treated on the same principles as males, with the exception that the dose of "9I4" does not exceed $0 \cdot 60$ gramme.

\section{GONORRHEA IN MALES}

In the acute stages daily irrigation is routine. The patient is taught to irrigate himself, and the usual solution employed is potassium permanganate of a strength not exceeding $\mathrm{r} / 8000$ The nozzle is of the ordinary Janet pattern, with a bluntly conical end ; the irrigator is of the Valentine pattern, and the height of the solution above the urethra must not exceed 3 feet 6 inches. The solution is allowed to flow into the bladder as soon as the sphincter can be persuaded to open, and the bladder then becomes the instrument by which the urethra is irrigated. I believe that it is a much more efficient 


\section{MANAGEMENT OF SYPHILIS AND GONORRHCA}

irrigator than the vessel which is called by that name. Such complications as epididymitis, which are feared by those who restrict the irrigation to the anterior urethra until the posterior urethra is so much infected that the secretion from it makes the second glass of urine turbid, do not occur any more frequently under this system than when the irrigation is confined to the anterior urethra. The important conditions are that the strength of the lotion and the height from which it is delivered shall not exceed those mentioned. As a variant from potassium permanganate, acriflavine, I/5000, or silver nitrate, $\mathrm{I} / \mathrm{IO}, 000$, is sometimes used, and if secondary infection should occur, either acriflavine, silver nitrate, I/I0,000, or mercury oxycyanide, $\mathrm{I} / 4000$ to $\mathrm{I} / 8000$, is preferred. Medicines are prescribed only when it is clear that the patient requires a sedative diuretic. Instead, bland drinks in large quantities are recommended. The patient receives a card on which some simple rules of life are printed. Mainly, these tell him to avoid alcohol, condiments, watercress, strawberries, and asparagus, and to live chiefly on such milky and starchy foods as bread, jam, milk puddings, vegetables and fruit, with restricted meat ; to avoid the other sex, love stories, and suggestive pictures; to wear a good suspensory bandage, and take advice on the question of exercise ; also not to worry, and not to examine himself daily.

The patient is seen by a medical officer once a week, and when the time comes for application of the tests of cure, or for the institution of treatment for chronic gonorrhœa, an appointment is made with him to attend at some time when the department is not busy. $\mathrm{He}$ is then examined in careful detail to determine what, if any, further treatment is required. The further treatment is designed to secure drainage of any foci of infection which remain unhealed. Massage is employed if the posterior urethra and the prostate are infected, and dilatation and the passage of curved sounds are sometimes supplemented by cauterisation of infected follicles when the anterior urethra is the seat of the trouble.

Vaccines are employed fairly freely, being generally gonococcal mixed with the organisms which are commonly found in the urethra in secondary infection.

In such complications as arthritis and epididymitis, vaccine treatment is supplemented by intravenous injec- 


\section{BRITISH JOURNAL OF VENEREAL DISEASES}

tions of electrargol in doses ranging usually from 5 to Io c.c. every other day.

Experience of diathermy has fully justified the claims of Cumberbatch and Robinson as to its good effects in epididymitis. In prostatitis and in all metastatic complications diathermy applied to the prostate and vesicles has proved of great value in shortening the attack.

\section{Gonorrhea in Females}

In all cases, whatever the complaint, a thorough clinical examination of the genito-urinary organs is carried out, including palpation of the uterus and tubes. Specimens for cultural and microscopical examination are taken from the urethra and cervical canal, and the blood is examined for both syphilitic and gonococcal serum reactions. These examinations are repeated, except in the case of the serum test for syphilis, as soon as possible after each menstrual period. The following systematic treatment is carried out in all non-pregnant cases of gonorrhœa, the patient attending daily if possible for the applications to be made by a trained nurse.

(I) Irrigation of the urethra, employing a glass catheter, with saturated solution of bicarbonate of soda, followed by-.

Chloramine T., I oz.

Sodium chloride, I oz.

Water to 24 ozs.

Diluted further to I in 40 just before use.

(2) Cleansing of the cervical canal by swabbing with saturated solution of bicarbonate of soda, followed by Chloramine $\mathrm{T}$. solution.

(3) Irrigation of the cervical canal with saturated solution of bicarbonate of soda, followed by Chloramine $\mathrm{T}$. solution as above, employing for the purpose a special backflow metal instrument with spiral grooves, to give the irrigation solution a spin.

(4) Packing of the vagina with I yard of gauze folded longitudinally into three and soaked in the following :-

First week, glycerine of borax

Second week, glycerine of anosol

Third week, glycerine of euca-

Io per cent.

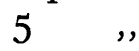

lyptus 


\section{MANAGEMENT OF SYPHILIS AND GONORRHCA}

Or with a I per cent. solution of Di-chloramine $\mathrm{T}$. in 20 per cent. chlorinated eucalyptus oil and 80 per cent. chlorinated paraffin.

Fourth week, the same as the first.

Fifth week, the same as the second, and so on.

In some cases a wick of gauze soaked in one of the above glycerines is pushed into the cervical canal and left for twenty-four hours.

(5) Injection of detoxicated gonococcal vaccine in the following doses :-

\begin{tabular}{|c|c|c|c|c|c|}
\hline First week & & & & $\mathrm{I}, \mathrm{OOO} \mathrm{I}$ & illions \\
\hline Second week & & & & $\mathrm{I}, 000$ & , \\
\hline Third week & & & & 2,000 & ," \\
\hline Fourth week & & & & 3,000 & , \\
\hline Fifth week . & - & • & & 3,000 & , \\
\hline Sixth week . & - & - & & 5,000 & , , \\
\hline Seventh week & & & & 5,000 & , \\
\hline Eighth week & & & & 5,000 & ,, \\
\hline
\end{tabular}

If secondary infection is discovered the vaccine is gonococcal mixed with secondary organisms.

When patients do not clear up, and it is obvious that the body of the uterus is infected, the treatment recommended by Hobbs is adopted. A soft rubber catheter (No. 3 English) is passed into the uterus without dilatation, and some liniment of iodine diluted to I in 8 with glycerine is gently syringed through the catheter, which is removed after six hours.

The treatment of pregnant women is similar to the above, except that applications to the cervical canal and vaginal packing are omitted. Instead, the vagina and vaginal surface of the cervix are swabbed with the medicated glycerines mentioned above.

The principle of this treatment is to raise the resistance and promote drainage, so as to assist the patient to rid herself of the infection. Caustic applications are not employed, as it is believed that they interfere with drainage by coagulating the surface tissues, and that necrosed tissues resulting from cauterisations are a favourable medium for the growth of secondary organisms. At the same time no remedy has yet been discovered which will penetrate the tissues and reach the gonococci in sufficient strength to destroy them. 


\section{BRITISH JOURNAL OF VENEREAL DISEASES}

The above treatment was introduced as an experimental measure over four years ago with the intention that it should be abandoned, or modified, if after three months' trial the results were found to be worse than those following any of the multitude of caustic forms of treatment which had preceded it. It is agreed in the department that the results have been very much better.

Diathermy is also employed in a number of cases, but is at present in the experimental stage, as it is felt that the electrodes and the method of testing the dosage require improvement before results become constant.

\section{Vulvo-Vaginitis in Female Children}

For children who are not given to screaming and struggling the following has been adopted in place of the routine treatment followed by most of those who have the medical care of patients with this very troublesome complaint. It is based on the principle that the douching and swabbing which are commonly employed fail to reach gonococci in the higher parts of the vagina, and that it is generally these which are responsible for the frequent relapses which have broken many hearts.

The largest cannula of an aero-vaginoscope which the hymen will allow is passed to the top of the vagina. The vaginoscope is an aero-urethroscope fitted with larger cannulas, such as 28, 30, 32, and 34 French. After the introducer has been removed and the parts swabbed with wool on urethroscopic canes, the inspecting eyepiece and lamp are fitted, and the vagina is inflated. This opens out all the folds, and a perfect view of the cervix and vaginal fornices is obtained. The eye-piece is then removed, and the whole of the upper portion of the vagina is painted with freshly-made mercurochrome 220, in a strength of 25 per cent. The painting is continued until it is verified by inspection under air inflation that the whole surface has been treated. If the urethra is infected, it is also painted with mercurochrome, which, curiously, seems to cause no great irritation of this canal. If rectal infection exists, it is treated by douching in the ordinary way. The treatment through the vaginoscope is repeated about once each week. It has so far given most encouraging results in a number of cases. In others it has failed, and in these there has been evidence of 


\section{MANAGEMENT OF SYPHILIS AND GONORRHEA}

cervical infection. It is mentioned here, though it has not been successful in all cases, because it is safe and seems at least to be based on common-sense principles. For douching the vagina I have found quite useful an instrument which I employ for cleansing the anterior urethra when investigating cases of chronic gonorrhœa in males. It is a straight, hollow instrument, with holes at its very end and a deep furrow down one side which serves to conduct the returning fluid. A hollow cone fitted with an outflow pipe slides over the stem of the instrument and plugs the opening of the canal, being irrigated in such a way that the return flow is conducted into a vessel. 